\title{
A General Model for Periodic Chemical Production Scheduling
}

Yaqing Wu, Christos T. Maravelias

${ }^{1}$ Department of Chemical and Biological Engineering, University of Wisconsin-Madison, USA

\section{Supplementary Information}

\section{Single-stage example}

Table S1-1. Max. inventory level $(\mathrm{kg})$, inventory cost $(\$ /(\mathrm{kg} \cdot \mathrm{h}))$, and demand $(\mathrm{kg} / \mathrm{h})$

\begin{tabular}{llllll}
\hline & $\mathrm{A}$ & $\mathrm{B}$ & $\mathrm{C}$ & $\mathrm{D}$ & $\mathrm{E}$ \\
\cline { 2 - 6 } Max. inventory & Unlimited & Unlimited & Unlimited & Unlimited & Unlimited \\
Inventory cost & 1 & 1 & 1 & 1 & 1 \\
Demand & 0.5 & 1 & 0.5 & 0.5 & 1 \\
\hline
\end{tabular}

Table S1-2. Processing time (h) and cost (\$) (given in parentheses)

\begin{tabular}{llll}
\hline & U1 & U2 & U3 \\
\hline PA & $3(10)$ & $3(10)$ & $3(10)$ \\
PB & $6(10)$ & $6(10)$ & $6(10)$ \\
PC & $5(10)$ & $5(10)$ & $5(10)$ \\
PD & $5(10)$ & $5(10)$ & $5(10)$ \\
PE & $5(10)$ & $5(10)$ & $5(10)$ \\
\hline
\end{tabular}

Table S1-3. Unit capacities (kg)

\begin{tabular}{llll} 
& $\mathrm{U} 1$ & $\mathrm{U} 2$ & $\mathrm{U} 3$ \\
\cline { 2 - 4 } Min. capacity & 0 & 0 & 0 \\
Max. capacity & 15 & 15 & 15
\end{tabular}

Max. capacity $15 \quad 15 \quad 15$

\section{Mixed-process example}

Table S2-1. Max. inventory level $(\mathrm{kg})$ and inventory cost $(\$ /(\mathrm{kg} \cdot \mathrm{h}))$

\begin{tabular}{llllllllll}
\hline & IA1 & IB1 & IC1 & IA2 & IB2 & IC2 & A & B & C \\
\cline { 2 - 9 } Max. inventory & 300 & 300 & 300 & 300 & 300 & 300 & 300 & 300 & 300 \\
Inventory cost & 0.5 & 0.5 & 0.5 & 0.1 & 0.1 & 0.1 & & & \\
\hline
\end{tabular}

Table S2-2. Processing time (h) and cost (\$) (given in parentheses) of batch tasks

\begin{tabular}{llll}
\hline & PIA & PIB & PIC \\
\cline { 2 - 4 } Batch1 & $14(20)$ & $10(20)$ & $8(20)$ \\
Batch2 & $14(20)$ & $11(20)$ & $9(20)$ \\
\hline
\end{tabular}


Table S2-3. Min/max processing rate $(\mathrm{kg} / \mathrm{h}$ ) and cost $(\$ / \mathrm{h})$ (given in parentheses) of continuous tasks

\begin{tabular}{lllllll}
\hline & PIA2 & PIB2 & PIC2 & PA & PB & PC \\
\cline { 2 - 7 } PFR1 & $5 / 23.3(0.5)$ & $5 / 17.5(0.5)$ & $5 / 17.5(0.5)$ & & & \\
PFR2 & & & & $5 / 23.3(0.5)$ & $5 / 17.5(0.5)$ & $5 / 17.5(0.5)$ \\
\hline
\end{tabular}

\begin{tabular}{lll}
\multicolumn{3}{c}{ Table S2-4. Unit capacities $(\mathrm{kg})$} \\
\cline { 2 - 2 } Match1 & Batch2 \\
\cline { 2 - 3 } Min. capacity & 50 & 50 \\
Max. capacity & 70 & 70 \\
\hline
\end{tabular}

Table S2-1. Changeover time (h)

\begin{tabular}{llll}
\hline & PIA2 & PIB2 & PIC2 \\
\cline { 2 - 4 } PIA2 & & 6 & 12 \\
PIB2 & 6 & & 6 \\
PIC2 & 12 & 6 & \\
\hline
\end{tabular}

\section{Multi-stage example}

Table S3-1. Max. inventory level (kg), inventory cost $(\$ /(\mathrm{kg} \cdot \mathrm{h}))$, demand $(\mathrm{kg} / 24 \mathrm{~h})$, and price $(\$ / \mathrm{kg})$

\begin{tabular}{lllllll}
\hline \multirow{3}{*}{ Max. inventory } & IA1 & IB1 & IC1 & ID1 & IE1 & IF1 \\
\cline { 2 - 7 } Inventory cost & 0.5 & Unlimited & Unlimited & Unlimited & Unlimited & Unlimited \\
\cline { 2 - 7 } & $\mathrm{A}$ & $\mathrm{B}$ & $\mathrm{C}$ & $\mathrm{D}$ & $\mathrm{E}$ & $\mathrm{F}$ \\
\cline { 2 - 7 } Max. inventory & Unlimited & Unlimited & Unlimited & Unlimited & Unlimited & Unlimited \\
Demand & 3.6 & 2.4 & 6 & 3.6 & 3.6 & 6 \\
Price & 55 & 65 & 80 & 60 & 50 & 60 \\
\hline
\end{tabular}

Table S3-2. Processing time (h) and cost (\$) (given in parentheses)

\begin{tabular}{lllllll}
\hline & U1 & U2 & U3 & U4 & U5 & U6 \\
\cline { 2 - 7 } PA1 & $5(5)$ & $5(5)$ & $4(5)$ & & & \\
PA2 & & & & $9(5)$ & $7(5)$ & $7(5)$ \\
PB1 & $5(5)$ & $5(5)$ & $5(5)$ & & & \\
PB2 & & & & $9(5)$ & $7(5)$ & $7(5)$ \\
PC1 & $6(5)$ & $4(5)$ & $5(5)$ & & & \\
PC2 & & & & $9(5)$ & $7(5)$ & $7(5)$ \\
PD1 & $5(5)$ & $5(5)$ & $5(5)$ & & & \\
PD2 & & & & $7(5)$ & $9(5)$ & $8(5)$ \\
PE1 & $4(5)$ & $5(5)$ & $4(5)$ & & & \\
PE2 & & & & $8(5)$ & $9(5)$ & $7(5)$ \\
PF1 & $4(5)$ & $5(5)$ & $5(5)$ & & & \\
PF2 & & & & $8(5)$ & $7(5)$ & $9(5)$ \\
SA & $2(2)$ & $2(2)$ & $2(2)$ & $2(2)$ & $2(2)$ & $2(2)$ \\
SB & $1(2)$ & $1(2)$ & $1(2)$ & $1(2)$ & $1(2)$ & $1(2)$ \\
SC & $3(2)$ & $3(2)$ & $3(2)$ & $3(2)$ & $3(2)$ & $3(2)$ \\
SD & $3(2)$ & $3(2)$ & $3(2)$ & $3(2)$ & $3(2)$ & $3(2)$ \\
SE & $2(2)$ & $2(2)$ & $2(2)$ & $2(2)$ & $2(2)$ & $2(2)$ \\
SF & $3(2)$ & $3(2)$ & $3(2)$ & $3(2)$ & $3(2)$ & $3(2)$ \\
\hline
\end{tabular}


Table S3-3. Production tasks and the corresponding setup tasks

\begin{tabular}{lllllll}
\hline \multirow{3}{*}{ Setup tasks } & PA & PB & PC & PD & PE & PF \\
\cline { 2 - 6 } & SA & SB & SC & SD & SE & SF \\
\hline
\end{tabular}

Table S3-4. Unit capacities (kg)

\begin{tabular}{llllll}
\hline & $\mathrm{U} 1$ & $\mathrm{U} 2$ & $\mathrm{U} 3$ & $\mathrm{U} 4$ & $\mathrm{U} 5$ \\
\cline { 2 - 6 } Min. capacity & 1.2 & 1 & 1.4 & 1.4 & 1.6 \\
Max. capacity & 6 & 5 & 7 & 7 & 8 \\
\hline
\end{tabular}

\section{Network example}

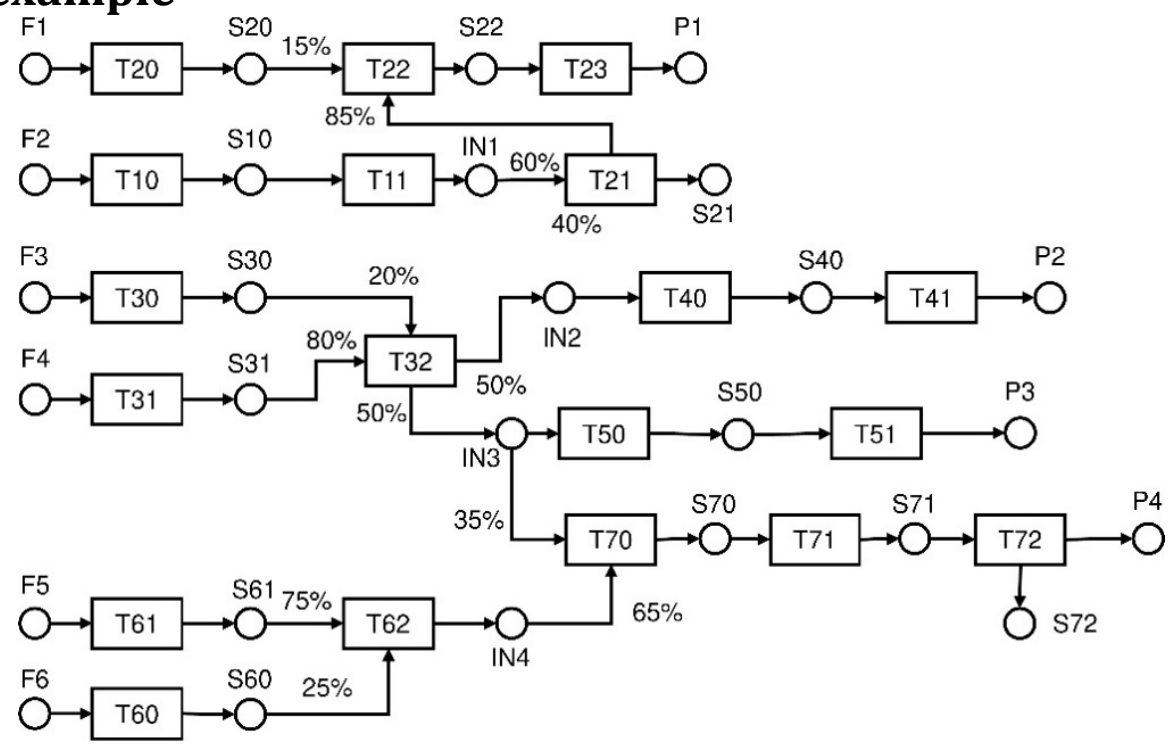

Fig. S1 The network process.

Table S4-1. Max. inventory level $(\mathrm{kg})$ and price $((\$) / \mathrm{kg})$

\begin{tabular}{lllllllllllll}
\hline \multirow{3}{*}{$\begin{array}{l}\text { Max. } \\
\text { inventory }\end{array}$} & S20 & S10 & S30 & S31 & S61 & S60 & IN1 & IN2 & IN3 & IN4 & S22 & S21 \\
\cline { 2 - 12 } & 500 & 0 & 1000 & 1000 & 1000 & 0 & 2500 & 2500 & 2500 & 2500 & 0 & 50 \\
\cline { 2 - 12 } $\begin{array}{l}\text { Max. } \\
\begin{array}{l}\text { inventory } \\
\text { Price }\end{array}\end{array}$ & 0 & 0 & 50 & 0 & 0 & 0 & 500 & 125 & 1250 & 1250 & 1250 & 1250 \\
\hline
\end{tabular}


Table S4-2. Processing time (h) and cost (\$) (given in parentheses)

\begin{tabular}{|c|c|c|c|c|c|c|c|c|}
\hline & $\mathrm{U} 1$ & U2 & U3 & U4 & U5 & U6 & U7 & U8 \\
\hline T20 & & & & & $5(20)$ & & & \\
\hline T10 & $4(10)$ & & & & & & & \\
\hline T30 & & & & $2(10)$ & & & & \\
\hline T31 & & & $2(15)$ & & & & & \\
\hline T61 & & & & & & $6(20)$ & & \\
\hline T60 & & & & $3(12)$ & & & & \\
\hline T22 & & & & & & & $2(25)$ & \\
\hline T11 & & & & & & & $3(30)$ & \\
\hline T32 & & $4(8)$ & & & & & & \\
\hline T62 & & & & & & & & $2(12)$ \\
\hline T23 & & & & $3(14)$ & & & & \\
\hline $\mathrm{T} 21$ & & & & & & & & \\
\hline $\mathrm{T} 40$ & & & & & 3 (18) & & & \\
\hline T50 & & & & & $2(25)$ & & & \\
\hline T70 & & & & & & $3(16)$ & & \\
\hline $\mathrm{T} 41$ & & $2(6)$ & & & & & & \\
\hline T51 & & $2(10)$ & & & & & & $2(10)$ \\
\hline T71 & & & & & & & & $2(8)$ \\
\hline T72 & & & $1(16)$ & & & & & $1(15)$ \\
\hline
\end{tabular}

Table S4-3. Unit capacities (kg)

\begin{tabular}{lllllllll}
\hline & U1 & U2 & U3 & U4 & U5 & U6 & U7 & U8 \\
\cline { 2 - 9 } Min. capacity & 1.2 & 1 & 1.4 & 1.4 & 1.6 & 1.2 & 1.4 & 1.6
\end{tabular}

$\begin{array}{lllllllll}\text { Max. capacity } & 6 & 5 & 7 & 7 & 8 & 6 & 7 & 8\end{array}$

\section{Model statistics}

Table S5-1. Model statistics

\begin{tabular}{lllll}
\hline & Subsection & Subsection & Subsection & \multirow{2}{*}{ Subsection 5.1.3 } \\
\cline { 2 - 5 } Constraints & 5.1 .1 & $5.1 .2(10 \mathrm{~h}$ period $)$ & $5.1 .2(26 \mathrm{~h}$ period) & \\
Discrete variables & 109 & 326 & 774 & 774 \\
Continuous & 109 & 269 & 269 \\
variables & 217 & 167 & 407 & 407 \\
Obj. values & 207.5 & 192.5 & 493 & 500 \\
CPU & 1 & 1 & 278 & 11 \\
Optimality gap & 0 & 0 & 0 & 0 \\
\cline { 2 - 5 } & Subsection & Subsection & Subsection & Subsection 5.3.2 \\
\cline { 2 - 5 } Constraints & 5.1 .4 & 5.2 & 5.3 .1 & 8650 \\
Discrete variables & 309 & 2318 & 12047 & 2561 \\
Continuous & 496 & 1161 & 10816 & 5084 \\
variables & 497 & 2506 & 17408 & 14035 \\
Obj. values & 563 & 1478.5 & 23712 & 1800 \\
CPU & 13 & 58 & 3600 & 2.09 \\
Optimality gap & 0 & 0 & 4.18 & \\
\hline
\end{tabular}

The units of obj. values, CPU, and optimality gap are $\$$, s, and $\%$, respectively. 